\title{
PROGESOS DE LECTURA EN ESTUDIANTES UNIVERSITARIOS
}

\author{
Alejandro David Garcia \\ Docente Programa de Psicopedagogia \\ Facultad de Educación \\ adavidgarciav@hotmail.com \\ Luz Ángela Ortiz \\ Licenciada en Lengua Castellana \\ Universidad Surcolombiana \\ eclipsisnaun@hotmail.com
}

\section{RESUMEN}

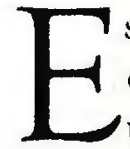

sta es una investigación sobre los procesos de lectura que realizan los estudiantes universitarios (primer semestre) de la Universidad Surcolombiana, con el fin de detectar la manera como elaboran predicciones con la lectura, activan saber previo, plantean objetivos antes de la lectura; así mismo, si hacen monitoreo durante la lectura y verifican hipótesis o predicciones, elaboran inferencias, entre otras; y si hacen evaluación de la lectura cuando terminan el texto, en este caso si terminan de confirman sus hipótesis y predicciones y generan nuevas inquietudes con la lectura y si son conscientes de lo aprendido.

Palabras claves: proceso de lectura, estrategias de lectura, hipótesis, predicción, lectura, prelectura, postlectura.

\section{INTRODUCCIÓN}

El trabajo aborda la manera como un grupo de estudiantes, del primer semestre de Licenciatura en educación básica con énfasis en humanidades y Lengua castellana, de la Universidad Surcolombiana realizan sus procesos de lectura. Para ello, se elaboró una entrevista que analiza dos aspectos de los lectores: su contexto como lector que busca auscultar los saberes previos, conocimientos y hábitos que tienen sobre la lectura; el otro aspecto analiza, cómo llevan a cabo estos estudiantes sus procesos de lectura (prelectura, lectura, postlectura) y las posibles estrategias o técnicas de lectura que emplean al abordar un texto.

La necesidad de estudiar los procesos de lectura se debe a la precaria formación lectora que presentan históricamente los estudiantes cuando

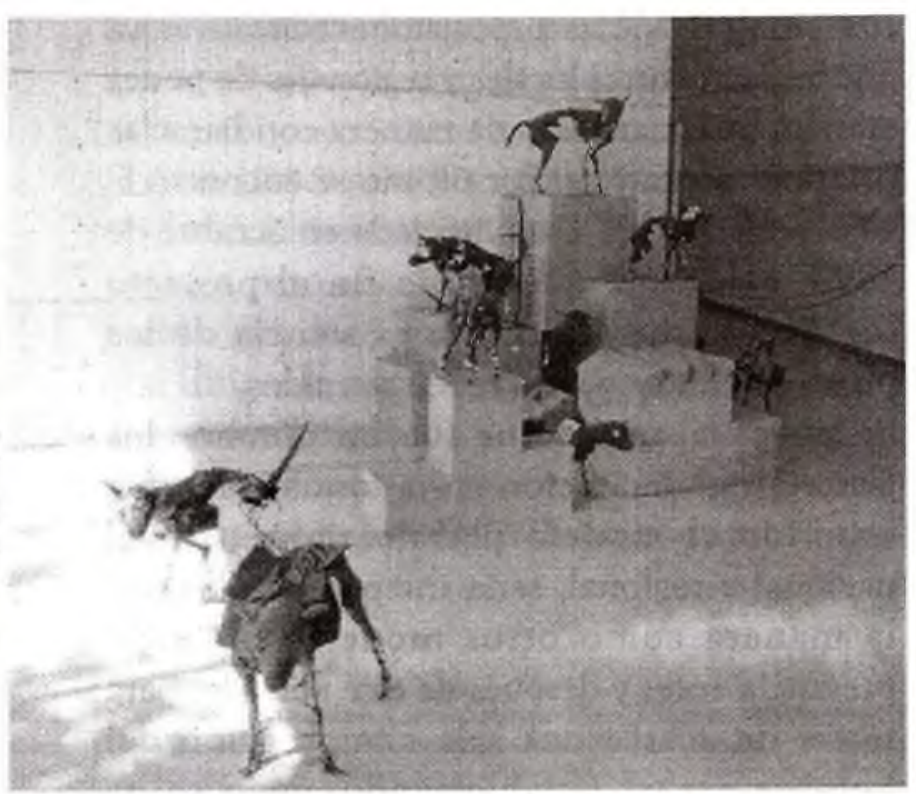

MIGUEL KUAN : "PERRO IDOS..." INSTALACIÓN 2009 -Pitalito 
ingresan a la universidad, pues la educación colombiana, se ha caracterizado por los bajos niveles de comprensión lectora que presentan los estudiantes de la básica secundaria; más adelante, aquellos que ingresan a la universidad, presentan deficiencia en la lectura y vicios lectores, porque han sido educados a partir de una lectura mecánica y literal. La incapacidad para mantener la observación y atención sostenida, el desconocimiento de la función de los paratextos en la comprensión lectora, la identifican de forma incompleta de las ideas del texto, la falta de competencias complejas que le permitan pasar de un significado explicito a uno implicito del texto, el desconocimiento del sentido de las palabras y el escaso conocimiento de las estructuras textuales, la precariedad en la escritura, las estrategias lectoras inadecuadas, los procesos lectores fragmentados, la preponderancia de la comprensión lectora literal sobre la inferencial y critica, son todos un impedimento para desarrollar el pensamiento complejo propio de la universidad. Esto conlleva a la formación de lectores heterónomos, sin criterios y con hábitos mecánicos que irán a modelar socialmente lo que tradicionalmente se ha establecido.

Este es un bagaje previo con el que generalmente llega el estudiante a la universidad. Lo que evidencia el "choque" que genera el paso del colegio a la universidad, es un cambio que exige al estudiante unos niveles de comprensión lectora que desbordan la literalidad en su exigencia académica; cambios que demanda la asimilación de comprensión inferencial y critica para desarrollar competencias argumentativas, interpretativas $y$ propositivas, y que si el estudiante no lo asume se quede en lecturas superfluas que no le permiten apropiarse del conocimiento. Otro aspecto a considerar es que además de lo anterior, el estudiante ingresa a la universidad sin ese sentido de responsabilidad frente al estudio, por lo cual la lectura se convierte en un acto obligatorio y sin compromiso.
Teniendo en cuenta la descripción anterior, esta investigación pretende abordar los procesos de lectura que los estudiantes del primer semestre de lengua castellana realizan, con el fin de detectar las estrategias lectoras utilizadas por ellos.

Metodológicamente es una investigación descriptiva ya que pretende abordar la manera como los estudiantes llevan a cabo sus procesos de lectura en la universidad y se inscribe desde un paradigma cuantitativo y cualitativo; puesto que desarrolla inicialmente la puntualización, el registro de la información y luego es tabulada y trabajada por porcentajes e interpretada a la luz de la teoria. (Ver esquema 1)

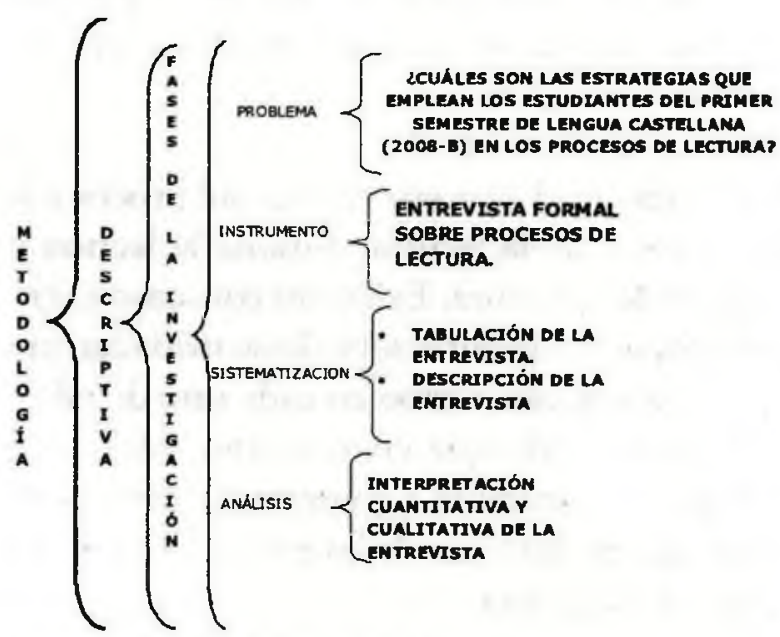

Esquema 1. Metodologia de la investigación

La interpretación de la información toma un enfoque descriptivo y hermenéutico, en tal sentido se tendrá información que se categorizará de acuerdo a los tres momentos en el proceso de la lectura: En la prelectura se tendrá en cuenta la formulación de hipótesis y predicciones de la lectura; durante la lectura se interpreta la confirmación y/o revaluación de hipótesis; en la postlectura se analiza la confirmación de predicciones y la elaboración de nuevas hipótesis en el abordaje del texto.

Una vez establecidas las categorias se validaran teóricamente con el fin de significar el proceso lector hecho por los estudiantes. 
El proceso de la lectura.

El proceso de la lectura es interno, inconsciente, del que no tenemos prueba hasta que nuéstras predicciones no se cumplen; es decir, hasta que comprobamos que en el texto no está lo que esperamos leer. Este proceso debe asegurar que el lector comprende el texto y que puede ir construyendo ideas sobre el contenido extrayendo de él aquello que le interesa. Esto sólo puede hacerlo mediante una lectura individual, precisa, que le permita avanzar y retroceder, que le permita detenerse, pensar, recapitular, relacionar la información nueva con el conocimiento previo que posee. Además deberá tener la oportunidad de plantearse preguntas, decidir qué es lo importante y qué es secundario. Es un proceso interno; que es imperioso enseñar.

Solé ${ }^{1}$, divide el proceso en tres subprocesos a saber: antes de la lectura, durante la lectura y después de la lectura. Existe un consenso entre todos los investigadores sobre las actividades que los lectores llevan a cabo en cada uno de ellos. Solé recomienda que cuando uno inicia una lectura se acostumbre a contestar las siguientes preguntas en cada uno de las etapas del proceso. Antes de la lectura.

¿Para qué voy a leer? (Determinar los objetivos de la lectura) ¿Qué sé de este texto? (Activar el conocimiento previo) ¿De qué trata este texto? ¿Qué me dice su estructura? (Formular hipótesis y hacer predicciones sobre el texto).

Durante la lectura.

¿Corresponde lo leido a lo supuesto inicialmente? ¿Qué dudas tengo del texto? ¿Cómo identifica en la lectura los planteamientos básicos del texto? Formular preguntas sobre lo leído con el fin de verificar hipótesis y predicciones y formular otras nuevas, si es preciso hacerlo.

Para llevar a cabo lo anterior, el lector relee partes confusas, consultar el diccionario, piensa en voz alta para asegurar la comprensión, crea imágenes mentales para visualizar descripciones vagas .

Después de la lectura.

¿Si haces un resumen del texto, con qué finalidad lo haces? ¿Cómo sabes que comprendiste lo leido? ¿Por qué crees que se te dificultó la comprensión de del texto? Comprobar que se cumplieron los objetivos de la lectura. Formular preguntas sobre lo leido con el fin de confirmar hipótesis y predicciones y formular nuevas preguntas que proyecten al lector a la búsqueda de otros conocimientos.

Algunas de las actividades que se dan en esta fase son: Hacer resúmenes, sintesis, utilizar organizadores gráficos, cuadros, mapas mentales y otras.

Comprensión lectora y educación.

Considerar la lectura como un proceso constructivo conlleva utilizar enfoques muy distintos de los que se han venido utilizando para desarrollar la comprensión lectora. Implica que ya nos se enseñarán más técnicas aisladas de comprensión y que se dejará de comprobar la comprensión lectora literal tal como se ha venido haciendo. Porque la lectura, no es decodificar palabras de un texto; contestar preguntas después de una lectura, o una simple identificación de palabras.

Pearson, Roehler, Dole y Duffy (1992)

'SOLE, Isahel. Estrategias de lectura. Editorial Graw. Barcelona. 1998. 
afirman que los lectores competentes poseen unas caracteristicas bien definidas, estas son:

- Utilizan el conocimiento previo para darle sentido a la lectura.

- Monitorean su comprensión durante todo el proceso de la lectura.

- Toman los pasos necesarios para corregir los errores de comprensión una vez se dan cuenta que han interpretado mal lo leido.

- Pueden distinguir lo importante en los textos que leen.

- Resumen la información cuando leen.

- Hacen inferencias constantemente durante y después de la lectura.

- Preguntan.

El proceso de lectura en los estudiantes universitarios.

A continuación se muestra el análisis de algunas preguntas realizadas a los estudiantes en la entrevista sobre los procesos de lectura.

¿Qué hace cuando empiezas una lectura? (Ver gráfica 1)

Gráfica 1.Pregunta 2 de la entrevista.

\section{¿QUÉ HACE CUANDO EMPIEZA UNA LECTURA?}

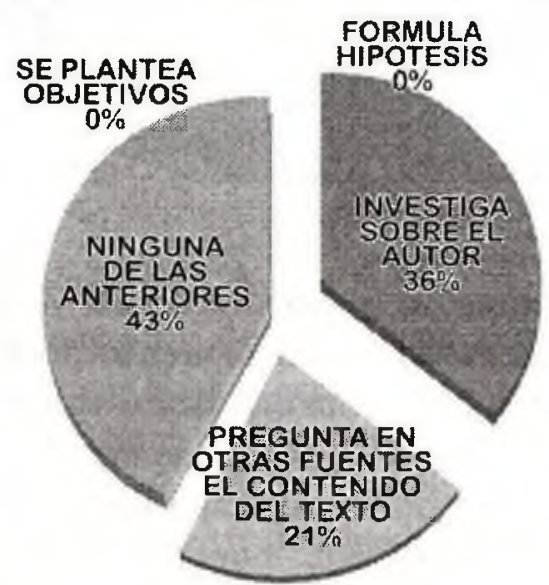

Fuente: Entrevista a estudiantes de Lengua Castellana I semestre. Cohorte 2008-2
Esta pregunta pretende indagar en los entrevistados si hacen una prelectura, si se formulan objetivos o hipótesis, si realizan otras actividades o ninguna de las anteriores; en otras palabras si se autodireccionan cuando empiezan a leer o no. Entendiendo autodirección como la existencia de un objetivo y la conciencia de que ese objetivo existe. $^{2}$

El análisis fue el siguiente: ningún estudiante se plantea objetivos o hipótesis de manera consciente en la lectura, el $43 \%$ no realiza ningún proceso mental a la hora de acercarse a un libro, por ejemplo: dos de los entrevistados (siete y trece) E7 dice que lo primordial antes de iniciar una lectura "busco un lugar adecuado en el cual pueda lograr una máxima concentración lectora, para así lograr un buen entendimiento del texto...", por su parte E13 dice "Mi estilo de leer es tomarlo y la lectura que sea libro fotocopia y lo comienzo a leer de una forma normal..." Eso quiere decir que este porcentaje le costará mucho más trabajo comprender un texto, puesto que leerán sin ningún objetivo definido, sin ningún problema a buscar.

Un $57 \%$ aparecen como lectores heterónomos, los cuales dependen de otras fuentes antes de acercarse a un texto. En el caso de los estudiantes pertenecientes al $21 \%$, que preguntan a otras personas o busca en internet, el resumen del texto; y el $36 \%$ que busca información sobre el autor; se tienen los siguientes argumentos: E1 opina que "antes de leer el libro investigo acerca del autor y de pronto en Internet reviso comentarios acerca del libro". En definitiva, así muchos autores o libros afirmen que todos los lectores (incluyendo los malos) cuentan con la estrategia de muestreo; no obstante, los resultados demuestran lo contrario, tal vez producto de la educación en secundaria, la cual

${ }^{2}$ PENAA GONZALEZ, Josefina. Las estrategias de lectura: su utilización en el aula. Educere articulos, año $4, n^{\circ} 11$, octubre - noviembre - diciembre, 2000 . PI60 
les pudo haber enseñado teorías, pero no les enseñó a pensar por sí mismos. Y el $100 \%$ de los entrevistados le dan poca importancia a sus propias expectativas sobre el texto, actuando como lectores pasivos en el proceso de lectura. ¿Das un vistazo general al texto? (Ver gráfica 2)

Gráfica 2. Pregunta 7 de la entrevista.

\section{¿DAS UN VISTAZO GENERAL AL TEXTO?}

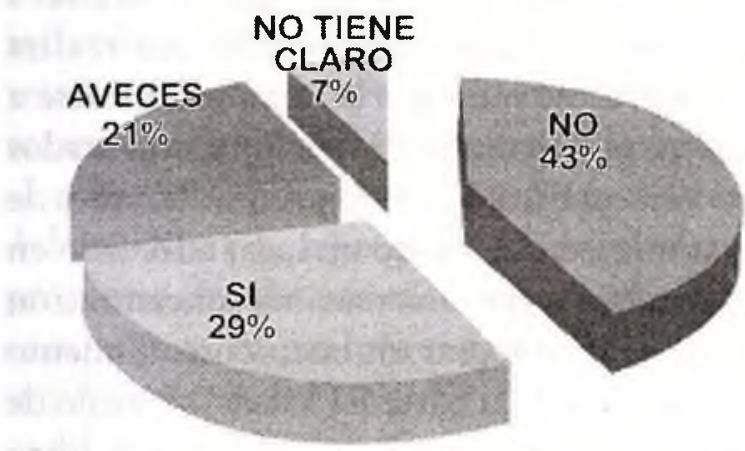

Fuente: Entrevista a estudiantes de Lengua Castellana I semestre. Cohorte 2008-2

Cuando un lector se aproxima a un texto lo primero que lo conecta a él son los paratextos. De acuerdo a Gérard Genette lo define como un elemento que ayuda al lector a introducirse en la lectura facilitando las primeras instrucciones sobre el contenido del libro. Por su parte, Gemma Lluch subdivide los paratextos en los que están fuera del libro, dentro del libro y los más visibles, en el análisis de esta pregunta se tendrán en cuenta los dos últimos.

Al analizar las respuestas de los estudiantes se pudo observar que el $72 \%$ de ellos dan un vistazo al libro, de los cuales un $36 \%$ se fijan en los nombres de los capitulos o subtitulos, y algunos otros en la introducción o prólogo. Para Gemma Lluch, estos pertenecen a los paratextos dentro del libro y adquieren importancia porque a partir de ellos el lector puede formular hipótesis predictivas sobre el texto; el $36 \%$, aparte de observar el nombre de los capitulos o subtitulos, se fijan en los paratextos más visibles, entre ellos, la tipografia y el número de páginas, aunque parezca algo superficial este tipo de elementos pueden influir notablemente en lector ya que si no tiene hábitos de lectura ver una gran cantidad de páginas con una letra menuda, lo puede desmotivar para seguir leyendo.

Volviendo al análisis general el $21 \%$ de los estudiantes entrevistados, afirman que lo importante al tomar un texto es el contenido o el fragmento que les interesa, no el libro en su totalidad y componentes externos. El 7\% restante no entendió la pregunta.

¿Cuando estás leyendo, tratas de suponer o anticipar lo que vas a encontrar en la lectura? (Ver gráfica 3)

\section{Gráfica 3. Pregunta 9 de la entrevista.}

\section{¿ CUANDO ESTAS LEYENDO TRATAS DE SUPONER O ANTICIPAR LO QUE VAS A ENCONTRAR EN LA LECTURA?}

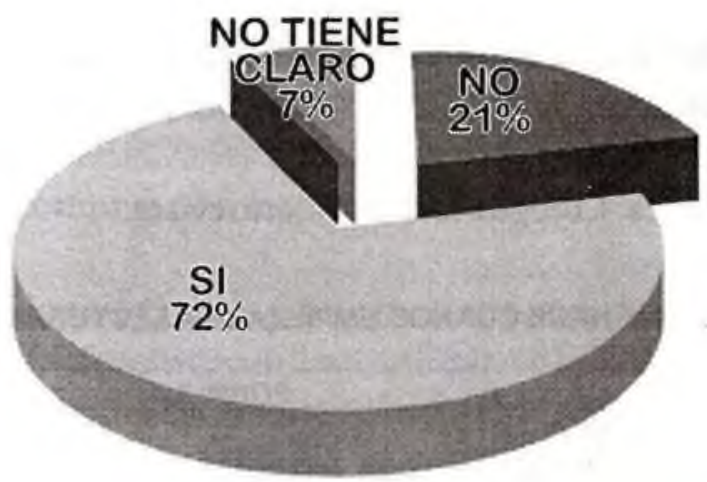

Fuente: Entrevista a estudiantes de Lengua Castellana I semestre. Cohorte 2008-2

En esta pregunta se pueden aclarar y contrastar otras preguntas anteriormente hechas, por ejemplo, el $72 \%$ de los estudiantes dan un vistazo general al texto, es decir que aquel $36 \%$ que observa los nombres de capitulos o subtitulos, introducciones, etc., pueden perfectamente predecir o formular hipótesis, tanto del capitulo como del texto en general, porque harian uso de sus conocimientos previos y teóricos 
adquiridos al dar el vistazo general al texto.

Por lo contrario, solamente un $29 \%$ trata de suponer $\mathrm{o}$ anticipar las acciones narrativas, $\mathrm{y}$ argumentan como, el entrevistado cuatro, que: E4 "Siempre se hace. Es algo voluntario, uno se monta la pelicula, se vuelve parte de la historia. Cuando la novela es abierta uno le da infinidad de finales y cuando es cerrado uno dice por qué no lo finalizo de tal forma. Voluntariamente uno va a ayudando a crear la historia". Y el 7\% faltante, está entre aquellos que no entendieron la pregunta, por tanto sus respuestas fueron incoherentes. Hasta ahora va el $36 \%$.

El $21 \%$ algunas veces trata de predecir, siempre y cuando sea de su interés el texto o de acuerdo al contexto de lectura.; y el $43 \%$ restante, está

Gráfica 4. Pregunta 20

\section{¿VERIFICAS QUE LOS OBJETIVOS}

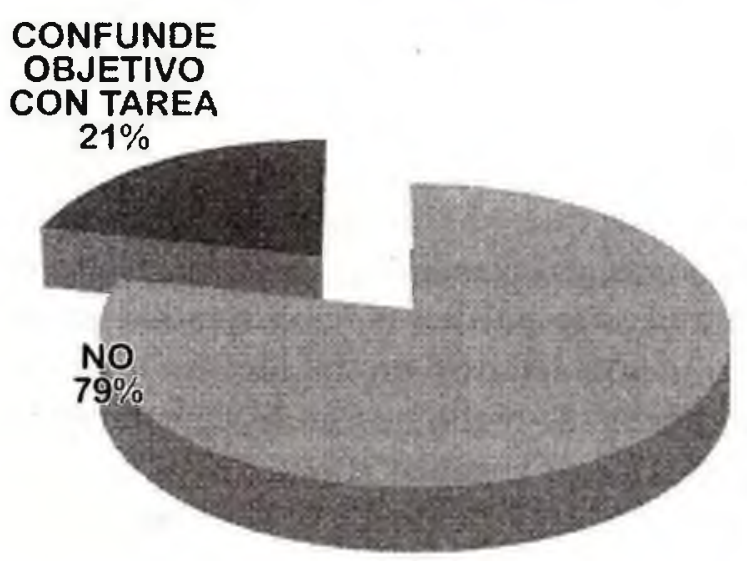

Estas dos preguntas, dejan observar la estrategia de confirmación y al mismo tiempo reafirmar la aplicación de la estrategia de muestreo, la cual permanece vigente durante el proceso de lectura; porque el lector debe autodireccionar y autocontrolar a partir de objetivos, hipótesis y estrategias la manera de abordar el texto. Al describir la primera pregunta, el $79 \%$ de los entrevistados no verifican objetivos porque no se los formulan al iniciar la lectura, y el $21 \%$ dividido entre aquellos que afirmaron sin dar argumentos y los que creen que no es necesario porque el texto lo da todo, por ejemplo el entrevistado siente dice lo siguiente: "E7: no, por lo general espero a que el texto me informe y me entregue conocimiento."

De nuevo se pueden percibir que los lectores están adiestrados en la lectura pasiva e irreflexiva, de modo que no se sienten capaces de suponer o formular supuestos, es decir que solamente un $29 \%$ de los lectores encuestados aplican la estrategia de predicción en la lectura de forma empirica.

¿Verificas que los objetivos planteados se hayan cumplido? y ¿los supuestos que hiciste antes y durante la lectura fueron confirmados finalmente?

\section{Gráfica 5. Pregunta 21}

\section{¿ LOS SUPUESTOS QUE HICISTE ANTES Y DURANTE LA LECTURA FUERON CONFIRMADOS FINALMENTE?}

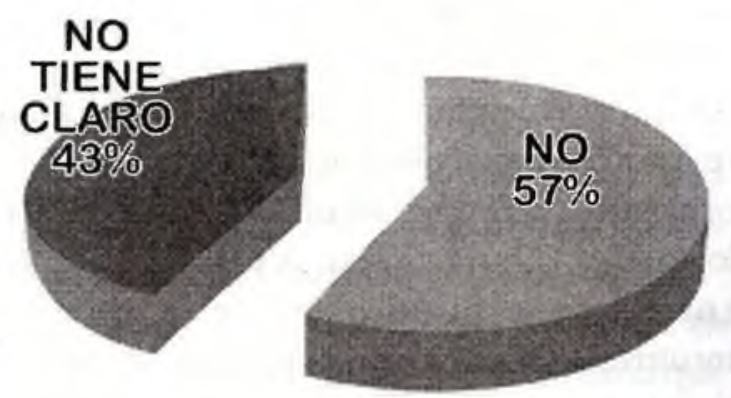

Fuente: Entrevista a estudiantes de Lengua Castellana I semestre. Cohorte 2008-2

afirman que verifican objetivos, pero sus respuestas dejan ver la confusión entre el término tarea y objetivo de lectura; por ejemplo: E9 contesta que "muchas veces el objetivo es hacer la sintesis y si la hago bien es porque el objetivo se cumplió, o en la nota o por el simple hecho de haber entendido ciertas cosas del texto". En cuanto a la segunda pregunta el $57 \%$ respondió no confirmar supuestos, el $43 \%$ aunque argumentan la respuesta dejan ver que 
no hacen supuestos de la lectura, por ejemplo: E1 dice: "Siempre lo hago, porque a veces uno tiene idea de una cosa y de pronto un texto te la plantea de otra forma...lo pone a uno en contradicción y lo pone a uno indagar a ver quién tiene la razón". Se puede afirmar con certeza que el $100 \%$ no hacen supuestos antes y durante la lectura. Esto se comprueba con la pregunta ¿Qué hace cuando empiezas una lectura? Alli ninguno de los estudiantes se formuló objetivos e hipótesis antes de la lectura. En conclusión si no hay una estrategia de muestreo, consciente o empirica, no puede haber una estrategia de confirmación y corrección.

1. Estrategias utilizadas por los estudiantes de primer semestre de Lengua castellana 2008-B:

${ }^{*}$ El $93 \%$ de los entrevistados, o sea trece estudiantes, no tienen conocimientos sobre estrategias de lectura de muestreo, predicción, inferencia, verificación y corrección. De este grupo, ocho estudiantes (57\%) desconocen el concepto como tal y cinco estudiantes (36\%) confunden estrategias con técnicas de lectura, aclarando que estas son de tipo más empírico que técnico. Sólo un estudiante (7\%) conoce el concierto de estrategias de lectura, Pero como se pudo comprobar más adelante, es un concepto que no ha asimilado, por lo tanto no lo aplica a la hora de leer. A pesar de esto, en la entrevista se pudo observar la aplicación intuitiva de la estrategia de predicción, en la cual se mantuvieron cuatro estudiantes (29\%) en la prelectura y durante la lectura no verifican los supuestos o predicciones iníciales.

2. Procesos de lectura utilizadas por los estudiantes de primer semestre de Lengua castellana 2008-B:

${ }^{\star}$ El $100 \%$ de los estudiantes no tienen un proceso de lectura, como se describe a continuación:

a) Antes de la lectura: En ese momento se aplica la estrategia de muestreo, inmersa en ella la caracteristica de autodirección.
$\mathrm{Al}$ analizar las respuestas de los entrevistados se pudo observar que diez estudiantes (72\%) dan un vistazo al libro, de los cuales cinco de ellos $(36 \%)$ se fijan en los nombres de los capitulos o subtítulos, y algunos otros en la introducción o prólogo, proceso importante porque a partir de ellos el lector puede formular predicciones sobre el texto; el 36\%, aparte de observar el nombre de los capítulos o subtítulos, se fijan en los paratextos más visibles, entre ellos, la tipografia y el número de páginas, Pero a pesar de hacer esta prelectura esto no significa que hagan predicciones sobre la lectura.

Lo cual se muestra cuando Diez estudiantes (71\%) no se preguntan de qué se trata el texto, pues ocho de ellos (57\%) esperan que el texto le trasmita todo, del mismo modo dependen del contenido que les brinde otras fuentes de información. Otros 2 estudiantes (14\%) no saben que se deben hacer estas preguntas. Eso permite observar que son lectores pasivos/ heterónomos y no se sienten capaces de preguntarse y elaborar significados del texto. Estos resultados repercuten más adelante cuando se observe en el momento después de la lectura, puesto que los estudiantes no podrán asegurar que verifican y corroborar sus supuestos, sencillamente porque no los hicieron. De nuevo se pueden percibir que los lectores están adiestrados en la lectura pasiva e irreflexiva, de modo que no se sienten capaces de suponer o formular supuestos, es decir que solamente un $29 \%$ de los lectores encuestados aplican la estrategia de predicción en la lectura de forma empírica.

\section{b) Durante la lectura:}

En este momento, el lector pone en uso las estrategias de inferencia, predicción $y$ confirmación o verificación de hipótesis (en un primer momento), dentro de ellas, sus respectivas caracteristicas como autodirección, autocontrol y flexibilidad. 
Durante la lectura, se pudo observar que diez estudiantes $(71 \%)$ no aplican estrategias de predicción, ní inferencia, esto conlleva a que no verifiquen hipótesis porque no se han formulado supuestos. En el caso de los cuatro entrevistados (29\% faltante) aplican la estrategia de predicción en la lectura de forma empirica cuando suponen o anticipan lo que van a encontrar en el texto, ese mismo número infieren el sentido implicito del texto, como sus planteamientos básicos, puesto que no siempre el planteamiento básico está explícito en él. No obstante, a la hora de verificar supuestos, de estos cuatro estudiantes solamente uno de ellos emplea la estrategia de verificación de hipótesis. Por otro lado, con respecto a las caracteristicas de autodirección, autocontrol y flexibilidad en las estrategias de lecturas, se puede deducir que únicamente cuatro estudiantes (29\%), se autodireccionan, es decir que cuando predicen dan un rumbo al texto, así la mayoria no verifiquen. La caracteristica de autocontrol y flexibilidad se hicieron evidentes por medio de las preguntas 11,12 y 13 , en las cuales se pudo observar que doce estudiantes $(86 \%)$ afirmaron en que releian el texto y buscaban ayuda para aclarar sus dudas, sin embargo se pudo confirmar que solamente tres de ellos (21\%) son conscientes de su falla y recurren a posibles soluciones, de manera intuitiva.

\section{c) Después de la lectura}

En este momento, el lector aplica las estrategias de confirmación o verificación de objetivos y supuestos, como también evalúa las estrategias por medio del monitoreo para luego corregir si es necesario y establecer los criterios de la comprensión lectora.

Se hizo evidente que seis entrevistados (43\%) hacen una verificación de la comprensión de lectura en un nivel muy literal y empírico, puesto que cuando resumen un texto este se circunscribe a los planteamientos básicos, ese mismo número de estudiantes realizan ayudas cognitivas como mapas conceptuales, cuadros sinópticos, entre otros, pero más por obligación que por probar su comprensión.

A consecuencia de esto, se pudo inferir también que los estudiantes no están seguros cuando comprenden un texto, por lo tanto nueve de ellos $(64 \%)$ no saben o no tienen clara la pregunta porque leen mecánicamente y acuden al texto directamente, en tal sentido, no se hacen preguntas o esperan encontrar la respuesta en otras fuentes (compañeros, profesores, internet, entre otros), lo cual los hace lectores heterónomos. Cinco estudiantes $(36 \%)$ se quedan en un nivel literal, es decir, desde lo que dice el texto como tal; dicho número hace parte de los seis estudiantes que realizan resúmenes, mapas conceptuales, cuadros sinópticos, entre otros, Pero, a pesar de ello, se puede asegurar que los entrevistados no aplican verificación de objetivos ni de hipótesis al finalizar la lectura, esto se confirmó mediante la pregunta 2,9 y 14 , en las cuales la mayoría de los estudiantes no se autodireccionaron a la hora de leer, ni aplicaron estrategias de muestreo y predicción, respectivamente. Hay que aclarar que es diferente verificar la compresión lectora a través de un resumen a que este sea producto de una estrategia de lectura consciente y voluntaria. Para finalizar, los lectores no se generan preguntas con respecto al mismo texto, sino que dejan ver su baja comprensión inferencial y crítica de la lectura, como es la situación de cinco de ellos que deben recurrir a otras fuentes para aclarar las dudas que tuvieron del texto; ocho de ellos $(57 \%)$ no lo hacen o no entendieron lo que se les preguntó, solamente uno de ellos (7\%) se genera preguntas desde el mismo texto, nuevamente, se puede decir que los lectores son heterónomos y pasivos, siempre esperan a que otras fuentes transmitan información porque no se sienten con las capacidades suficientes para construirlas por sí mismos. 
3. Formación lectora de los estudiantes de primer semestre de Lengua castellana 2008-B: Para empezar gran parte de los estudiantes de lengua castellana no tienen idea de qué es leer, otro está convencido que el libro lo transmite todo, como si ellos se considerasen una tabula rasa y un pequeño grupo de tres estudiantes consideran la lectura como un proceso interactivo, tienen el concepto pero no lo han asimilado en su práctica lectora, prueba de ello, el análisis de las entrevistas. Eso implica que si no conocen la lectura como proceso interactivo o transaccional dificilmente podrán tomar un papel activo como lectores, además de las dificultades que tendrán al acercarse a un texto desconociendo su función y la del texto dentro de la lectura, son lectores heterónomos que ignoran sus habilidades lectoras y se quedan en la literalidad y buscan a otros que aclaren sus dudas, lo que ellos sienten que no pueden hacer por si mismos.

A parte de esto, el análisis de las entrevistas dejó ver los siguientes problemas que tienen que ver con la formación lectora:

- Los estudiantes tienen problemas de comprensión de escucha, atención y observación.

- No cuentan con saberes previos suficientes que les permita comprender de manera adecuada textos complejos, científicos, filosóficos, políticos, epistemológicos, entre otros.

- No conocen los conceptos básicos como lectura, comprensión, interpretación o argumentación.
Bibliografia.

DUBOIS, Maria Eugenia. El proceso de lectura: de la teoria a la práctica, Aique. 1997. GOODMAN, Kenneth. (1982). "Proceso lector". En: Ferreiro, Emilia y Palacio, Margarita. Nuevas perspectivas de los procesos de lectura y escritura. México: Siglo XXI.

LERNER, Delia. Leer y escribir en la escuela: lo real, lo posible y lo necesario. F.C.E. México. 2003.

LLUCH, Gemma. Cómo analizamos relatos infantiles y juveniles. Editorial Norma. Bogotá 2004.

PEÑA GONZÁLEZ, Josefina. Las estrategias de lectura: su utilización en el aula. Educere artículos, año $4, \mathrm{n}^{\circ} 11$, octubre - noviembre diciembre, 2000.

PETIT, Michélle. Pero ¿Y qué buscan nuestros niños en sus libros? Conaculta-asolectura. Bogotá. 2004.

SOLÉ, Isabel. Estrategias de lectura. Editorial Grao. Barcelona. 1998.

Serie: Bajo Palabra (2001). Capitulos: Estrategias en el proceso lector; El autor y el lector en la comprensión lectora; El papel del texto en la comprensión; El texto escrito y el proceso de escritura. Departamento Audiovisuales, Universidad de Antioquia. 